\title{
Search for nonstandard neutrino interactions with IceCube DeepCore
}

M. G. Aartsen, ${ }^{2}$ M. Ackermann, ${ }^{52}$ J. Adams,${ }^{16}$ J. A. Aguilar, ${ }^{12}$ M. Ahlers, ${ }^{20}$ M. Ahrens, ${ }^{44}$ I. Al Samarai,,${ }^{25}$ D. Altmann, ${ }^{24}$ K. Andeen, ${ }^{33}$ T. Anderson, ${ }^{49}$ I. Ansseau, ${ }^{12}$ G. Anton, ${ }^{24}$ C. Argüelles, ${ }^{14}$ J. Auffenberg, ${ }^{1}$ S. Axani, ${ }^{14} \mathrm{H}$. Bagherpour,${ }^{16}$ X. Bai ${ }^{41}$ J. P. Barron, ${ }^{23}$ S. W. Barwick, ${ }^{27}$ V. Baum, ${ }^{32}$ R. Bay,${ }^{8}$ J. J. Beatty, ${ }^{18,19}$ J. Becker Tjus, ${ }^{11}$ K.-H. Becker, ${ }^{51}$ S. BenZvi, ${ }^{43}$ D. Berley, ${ }^{17}$ E. Bernardini, ${ }^{52}$ D. Z. Besson, ${ }^{28}$ G. Binder,${ }^{9,8}$ D. Bindig, ${ }^{51}$ E. Blaufuss, ${ }^{17}$ S. Blot, ${ }^{52}$ C. Bohm, ${ }^{44}$ M. Börner, ${ }^{21}$ F. Bos, ${ }^{11}$ D. Bose, ${ }^{46}$ S. Böser, ${ }^{32}$ O. Botner, ${ }^{50}$ E. Bourbeau, ${ }^{20}$ J. Bourbeau, ${ }^{31}$ F. Bradascio, ${ }^{52}$ J. Braun, ${ }^{31}$ L. Brayeur, ${ }^{13}$ M. Brenzke, ${ }^{1}$ H.-P. Bretz, ${ }^{52}$ S. Bron, ${ }^{25}$ J. Brostean-Kaiser, ${ }^{52}$ A. Burgman,,${ }^{50}$ T. Carver,${ }^{25}$ J. Casey, ${ }^{31}$ M. Casier, ${ }^{13}$ E. Cheung, ${ }^{17}$ D. Chirkin, ${ }^{31}$ A. Christov, ${ }^{25}$ K. Clark, ${ }^{29}$ L. Classen, ${ }^{36}$ S. Coenders, ${ }^{35}$ G. H. Collin, ${ }^{14}$ J. M. Conrad, ${ }^{14}$ D. F. Cowen, ${ }^{49,48}$ R. Cross ${ }^{43}$ M. Day, ${ }^{31}$ J. P. A. M. de André, ${ }^{22}$ C. De Clercq, ${ }^{13}$ J. J. DeLaunay, ${ }^{49}$ H. Dembinski, ${ }^{37}$ S. De Ridder, ${ }^{26}$ P. Desiati ${ }^{31}$ K. D. de Vries, ${ }^{13}$ G. de Wasseige, ${ }^{13}$ M. de With, ${ }^{10}$ T. DeYoung, ${ }^{22}$ J. C. Díaz-Vélez,${ }^{31}$ V. di Lorenzo, ${ }^{32}$ H. Dujmovic, ${ }^{46}$ J. P. Dumm, ${ }^{44}$ M. Dunkman, ${ }^{49}$ E. Dvorak, ${ }^{41}$ B. Eberhardt, ${ }^{32}$ T. Ehrhardt ${ }^{32}$ B. Eichmann, ${ }^{11}$ P. Eller, ${ }^{49}$ P. A. Evenson, ${ }^{37}$ S. Fahey,${ }^{31}$ A. R. Fazely, ${ }^{7}$ J. Felde, ${ }^{17}$ K. Filimonov, ${ }^{8}$ C. Finley, ${ }^{44}$ S. Flis ${ }^{44}$ A. Franckowiak, ${ }^{52}$ E. Friedman, ${ }^{17}$ T. Fuchs,${ }^{21}$ T. K. Gaisser, ${ }^{37}$ J. Gallagher, ${ }^{30}$ L. Gerhardt, ${ }^{9}$ K. Ghorbani, ${ }^{31}$ W. Giang, ${ }^{23}$ T. Glauch, ${ }^{1}$ T. Glüsenkamp, ${ }^{24}$ A. Goldschmidt, ${ }^{9}$ J. G. Gonzalez, ${ }^{37}$ D. Grant, ${ }^{23}$ Z. Griffith, ${ }^{31}$ C. Haack, ${ }^{1}$ A. Hallgren,${ }^{50}$ F. Halzen,${ }^{31}$ K. Hanson, ${ }^{31}$ D. Hebecker, ${ }^{10}$ D. Heereman, ${ }^{12}$ K. Helbing, ${ }^{51}$ R. Hellauer, ${ }^{17}$ S. Hickford,${ }^{51}$ J. Hignight, ${ }^{22}$ G. C. Hill, ${ }^{2}$ K. D. Hoffman, ${ }^{17}$ R. Hoffmann ${ }^{51}$ B. Hokanson-Fasig, ${ }^{31}$ K. Hoshina, ${ }^{31, *}$ F. Huang, ${ }^{49}$ M. Huber, ${ }^{35}$ K. Hultqvist, ${ }^{44}$ M. Hünnefeld, ${ }^{21}$ S. In, ${ }^{46}$ A. Ishihara, ${ }^{15}$ E. Jacobi,${ }^{52}$ G. S. Japaridze, ${ }^{5}$ M. Jeong, ${ }^{46}$ K. Jero, ${ }^{31}$ B. J. P. Jones, ${ }^{4}$ P. Kalaczynski, ${ }^{1}$ W. Kang ${ }^{46}$ A. Kappes, ${ }^{36}$ T. Karg ${ }^{52}$ A. Karle ${ }^{31}$ U. Katz, ${ }^{24}$ M. Kauer, ${ }^{31}$ A. Keivani, ${ }^{49}$ J. L. Kelley, ${ }^{31}$ A. Kheirandish, ${ }^{31}$ J. Kim, ${ }^{46}$ M. Kim, ${ }^{15}$ T. Kintscher, ${ }^{52}$ C. Kirby, ${ }^{14}$ J. Kiryluk, ${ }^{45}$ T. Kittler, ${ }^{24}$ S. R. Klein,,${ }^{9,8}$ G. Kohnen, ${ }^{34}$ R. Koirala ${ }^{37}$ H. Kolanoski ${ }^{10}{ }^{10}$ L. Köpke, ${ }^{32}$ C. Kopper ${ }^{23}$ S. Kopper, ${ }_{52}$ J. P. Koschinsky, ${ }^{1}$ D. J. Koskinen ${ }^{20}$ M. Kowalski, ${ }^{10,52}$ K. Krings, ${ }^{35}$ M. Kroll, ${ }^{11}$ G. Krückl, ${ }^{32}$ J. Kunnen, ${ }^{13}$ S. Kunwar, ${ }^{52}$ N. Kurahashi, ${ }^{40}$ T. Kuwabara, ${ }^{15}$ A. Kyriacou, ${ }^{2}$ M. Labare, ${ }^{26}$ J. L. Lanfranchi, ${ }^{49}$ M. J. Larson, ${ }^{20}$ F. Lauber $^{51}$ D. Lennarz, ${ }^{22}$ M. Lesiak-Bzdak, ${ }^{45}$ M. Leuermann, ${ }^{1}$ Q. R. Liu ${ }^{31}$ L. Lu, ${ }^{15}$ J. Lünemann, ${ }^{13}$ W. Luszczak, ${ }^{31}$ J. Madsen, ${ }^{42}$ G. Maggi, ${ }^{13}$ K. B. M. Mahn, ${ }^{22}$ S. Mancina, ${ }^{31}$ R. Maruyama ${ }^{38}$ K. Mase, ${ }^{15}$ R. Maunu, ${ }^{17}$ F. McNally, ${ }^{31}$ K. Meagher, ${ }^{12}$ M. Medici, ${ }^{20}$ M. Meier, ${ }^{21}$ T. Menne, ${ }^{21}$ G. Merino, ${ }^{31}$ T. Meures, ${ }^{12}$ S. Miarecki ${ }^{9,8}$ J. Micallef, ${ }^{22}$ G. Momente, ${ }^{32}$ T. Montaruli, ${ }^{25}$ R. W. Moore, ${ }^{23}$ M. Moulai, ${ }^{14}$ R. Nahnhauer, ${ }^{52}$ P. Nakarmi, ${ }^{47}$ U. Naumann,${ }^{51}$ G. Neer, ${ }^{22}$ H. Niederhausen, ${ }^{45}$ S. C. Nowicki, ${ }^{23}$ D. R. Nygren, ${ }^{9}$ A. Obertacke Pollmann, ${ }^{51}$ A. Olivas ${ }^{17}$ A. O'Murchadha, ${ }^{12}$ T. Palczewski ${ }^{9,8}$ H. Pandya, ${ }^{37}$ D. V. Pankova, ${ }^{49}$ P. Peiffer ${ }^{32}$ J. A. Pepper, ${ }^{47}$ C. Pérez de los Heros,${ }^{50}$ D. Pieloth, ${ }^{21}$ E. Pinat, ${ }^{12}$ M. Plum, ${ }^{33}$ P. B. Price, ${ }^{8}$ G. T. Przybylski ${ }^{9}$ C. Raab, ${ }^{12}$ L. Rädel, ${ }^{1}$ M. Rameez, ${ }^{20}$ K. Rawlins, ${ }^{3}$ I. C. Rea,${ }^{35}$ R. Reimann, ${ }^{1}$ B. Relethford, ${ }^{40}$ M. Relich, ${ }^{15}$ E. Resconi,${ }^{35}$ W. Rhode, ${ }^{21}$ M. Richman, ${ }^{40}$ S. Robertson, ${ }^{2}$ M. Rongen, ${ }^{1}$ C. Rott ${ }^{46}$ T. Ruhe ${ }^{21}$ D. Ryckbosch, ${ }^{26}$ D. Rysewyk ${ }^{22}$ T. Sälzer, ${ }^{1}$

S. E. Sanchez Herrera, ${ }^{23}$ A. Sandrock, ${ }^{21}$ J. Sandroos, ${ }^{32}$ M. Santander, ${ }^{47}$ S. Sarkar, ${ }^{20,39}$ S. Sarkar, ${ }^{23}$ K. Satalecka, ${ }^{52}$

P. Schlunder, ${ }^{21}$ T. Schmidt, ${ }^{17}$ A. Schneider, ${ }^{31}$ S. Schoenen, ${ }^{1}$ S. Schöneberg, ${ }^{11}$ L. Schumacher, ${ }^{1}$ D. Seckel,${ }^{37}$ S. Seunarine, ${ }^{42}$

J. Soedingrekso, ${ }^{21}$ D. Soldin, ${ }^{51}$ M. Song,${ }^{17}$ G. M. Spiczak, ${ }^{42}$ C. Spiering, ${ }^{52}$ J. Stachurska, ${ }^{52}$ M. Stamatikos, ${ }^{18}$ T. Stanev,${ }^{37}$

A. Stasik, ${ }^{52}$ J. Stettner, ${ }^{1}$ A. Steuer, ${ }^{32}$ T. Stezelberger, ${ }^{9}$ R. G. Stokstad, ${ }^{9}$ A. Stöß1, ${ }^{15}$ N. L. Strotjohann, ${ }^{52}$ T. Stuttard, ${ }^{20}$

G. W. Sullivan, ${ }^{17}$ M. Sutherland, ${ }^{18}$ I. Taboada, ${ }^{6}$ J. Tatar, ${ }^{9,8}$ F. Tenholt, ${ }^{11}$ S. Ter-Antonyan, ${ }^{7}$ A. Terliuk, ${ }^{52}$ G. Tešić, ${ }^{49}$

S. Tilav,${ }^{37}$ P. A. Toale, ${ }^{47}$ M. N. Tobin, ${ }^{31}$ S. Toscano, ${ }^{13}$ D. Tosi,${ }^{31}$ M. Tselengidou ${ }^{24}$ C. F. Tung, ${ }^{6}$ A. Turcati, ${ }^{35}$ C. F. Turley, ${ }^{49}$

B. Ty, ${ }^{31}$ E. Unger, ${ }^{50}$ M. Usner, ${ }^{52}$ J. Vandenbroucke, ${ }^{31}$ W. Van Driessche, ${ }^{26}$ N. van Eijndhoven, ${ }^{13}$ S. Vanheule, ${ }^{26}{ }^{23}$

J. van Santen, ${ }^{52}$ M. Vehring, ${ }^{1}$ E. Vogel, ${ }^{1}$ M. Vraeghe, ${ }^{26}$ C. Walck ${ }^{44}$ A. Wallace, ${ }^{2}$ M. Wallraff, ${ }^{1}$ F. D. Wandler, ${ }^{23}$

N. Wandkowsky, ${ }^{31}$ A. Waza, ${ }_{1}^{1}$ C. Weaver, ${ }^{23}$ M. J. Weiss, ${ }^{49}$ C. Wendt,${ }^{31}$ J. Werthebach, ${ }^{21}$ S. Westerhoff, ${ }^{31}$ B. J. Whelan, ${ }^{2}$ K. Wiebe, ${ }^{32}$ C. H. Wiebusch, ${ }^{1}$ L. Wille, ${ }^{31}$ D. R. Williams, ${ }^{47}$ L. Wills, ${ }^{40}$ M. Wolf, ${ }^{31}$ J. Wood, ${ }_{15}^{31}$ T. R. Wood, ${ }^{23}$ E. Woolsey, ${ }^{23}$ K. Woschnagg, ${ }^{8}$ D. L. Xu, ${ }^{31}$ X. W. Xu, ${ }^{7}$ Y. Xu, ${ }^{45}$ J. P. Yanez, ${ }^{23}$ G. Yodh, ${ }^{27}$ S. Yoshida, ${ }^{15}$ T. Yuan, ${ }^{31}$ and M. Zoll ${ }^{44}$

\section{(IceCube Collaboration)}

\author{
${ }^{1}$ III. Physikalisches Institut, RWTH Aachen University, D-52056 Aachen, Germany \\ ${ }^{2}$ Department of Physics, University of Adelaide, Adelaide 5005, Australia \\ ${ }^{3}$ Department of Physics and Astronomy, University of Alaska Anchorage, \\ 3211 Providence Dr., Anchorage, Alaska 99508, USA \\ ${ }^{4}$ Department of Physics, University of Texas at Arlington, 502 Yates St., Science Hall Rm 108, \\ Box 19059, Arlington, Texas 76019, USA \\ ${ }^{5}$ CTSPS, Clark-Atlanta University, Atlanta, Georgia 30314, USA \\ ${ }^{6}$ School of Physics and Center for Relativistic Astrophysics, Georgia Institute of Technology, \\ Atlanta, Georgia 30332, USA \\ ${ }^{7}$ Department of Physics, Southern University, Baton Rouge, Los Angeles 70813, USA
}


${ }^{8}$ Department of Physics, University of California, Berkeley, California 94720, USA

${ }^{9}$ Lawrence Berkeley National Laboratory, Berkeley, California 94720, USA

${ }^{10}$ Institut für Physik, Humboldt-Universität zu Berlin, D-12489 Berlin, Germany

${ }^{11}$ Fakultät für Physik und Astronomie, Ruhr-Universität Bochum, D-44780 Bochum, Germany

${ }^{12}$ Université Libre de Bruxelles, Science Faculty CP230, B-1050 Brussels, Belgium

${ }^{13}$ Vrije Universiteit Brussel (VUB), Dienst ELEM, B-1050 Brussels, Belgium

${ }^{14}$ Department of Physics, Massachusetts Institute of Technology, Cambridge, Massachusetts 02139, USA

${ }^{15}$ Department of Physics and Institute for Global Prominent Research, Chiba University, Chiba 263-8522, Japan

${ }^{16}$ Department of Physics and Astronomy, University of Canterbury, Private Bag 4800, Christchurch, New Zealand

${ }^{17}$ Department of Physics, University of Maryland, College Park, Maryland 20742, USA

${ }^{18}$ Department of Physics and Center for Cosmology and Astro-Particle Physics, Ohio State University, Columbus, Ohio 43210, USA

${ }^{19}$ Department of Astronomy, Ohio State University, Columbus, Ohio 43210, USA

${ }^{20}$ Niels Bohr Institute, University of Copenhagen, DK-2100 Copenhagen, Denmark

${ }^{21}$ Department of Physics, TU Dortmund University, D-44221 Dortmund, Germany

${ }^{22}$ Department of Physics and Astronomy, Michigan State University, East Lansing, Michigan 48824, USA

${ }^{23}$ Department of Physics, University of Alberta, Edmonton, Alberta T6G 2E1, Canada

${ }^{24}$ Erlangen Centre for Astroparticle Physics, Friedrich-Alexander-Universität Erlangen-Nürnberg, D-91058 Erlangen, Germany

${ }^{25}$ Département de physique nucléaire et corpusculaire, Université de Genève, CH-1211 Genève, Switzerland

${ }^{26}$ Department of Physics and Astronomy, University of Gent, B-9000 Gent, Belgium

${ }^{27}$ Department of Physics and Astronomy, University of California, Irvine, California 92697, USA

${ }^{28}$ Department of Physics and Astronomy, University of Kansas, Lawrence, Kansas 66045, USA

${ }^{29}$ SNOLAB, 1039 Regional Road 24, Creighton Mine 9, Lively, Ontario P3Y 1N2, Canada

${ }^{30}$ Department of Astronomy, University of Wisconsin, Madison, Wisconsin 53706, USA

${ }^{31}$ Department of Physics and Wisconsin IceCube Particle Astrophysics Center, University of Wisconsin, Madison, Wisconsin 53706, USA

${ }^{32}$ Institute of Physics, University of Mainz, Staudinger Weg 7, D-55099 Mainz, Germany

${ }^{33}$ Department of Physics, Marquette University, Milwaukee, Wisconsin 53201, USA

${ }^{34}$ Université de Mons, 7000 Mons, Belgium

${ }^{35}$ Physik-department, Technische Universität München, D-85748 Garching, Germany

${ }^{36}$ Institut für Kernphysik, Westfälische Wilhelms-Universität Münster, D-48149 Münster, Germany

${ }^{37}$ Bartol Research Institute and Department of Physics and Astronomy, University of Delaware, Newark, Delaware 19716, USA

${ }^{38}$ Department of Physics, Yale University, New Haven, Connecticut 06520, USA

${ }^{39}$ Department of Physics, University of Oxford, 1 Keble Road, Oxford OX1 3NP, UK

${ }^{40}$ Department of Physics, Drexel University, 3141 Chestnut Street, Philadelphia, Pennsylvania 19104, USA

${ }^{41}$ Physics Department, South Dakota School of Mines and Technology, Rapid City, South Dakota 57701, USA

${ }^{42}$ Department of Physics, University of Wisconsin, River Falls, Wisconsin 54022, USA

${ }^{43}$ Department of Physics and Astronomy, University of Rochester, Rochester, New York 14627, USA

${ }^{44}$ Oskar Klein Centre and Department of Physics, Stockholm University, SE-10691 Stockholm, Sweden

${ }^{45}$ Department of Physics and Astronomy, Stony Brook University, Stony Brook, New York 11794-3800, USA

${ }^{46}$ Department of Physics, Sungkyunkwan University, Suwon 440-746, Korea

${ }^{47}$ Department of Physics and Astronomy, University of Alabama, Tuscaloosa, Alabama 35487, USA

${ }^{48}$ Department of Astronomy and Astrophysics, Pennsylvania State University, University Park, Pennsylvania 16802, USA

${ }^{49}$ Department of Physics, Pennsylvania State University, University Park, Pennsylvania 16802, USA

${ }^{50}$ Department of Physics and Astronomy, Uppsala University, Box 516, S-75120 Uppsala, Sweden 
${ }^{51}$ Department of Physics, University of Wuppertal, D-42119 Wuppertal, Germany

${ }^{52}$ DESY, D-15738 Zeuthen, Germany

(Received 23 September 2017; published 12 April 2018)

\begin{abstract}
As atmospheric neutrinos propagate through the Earth, vacuumlike oscillations are modified by Standard Model neutral- and charged-current interactions with electrons. Theories beyond the Standard Model introduce heavy, TeV-scale bosons that can produce nonstandard neutrino interactions. These additional interactions may modify the Standard Model matter effect producing a measurable deviation from the prediction for atmospheric neutrino oscillations. The result described in this paper constrains nonstandard interaction parameters, building upon a previous analysis of atmospheric muon-neutrino disappearance with three years of IceCube DeepCore data. The best fit for the muon to tau flavor changing term is $\epsilon_{\mu \tau}=-0.0005$, with a $90 \%$ C.L. allowed range of $-0.0067<\epsilon_{\mu \tau}<0.0081$. This result is more restrictive than recent limits from other experiments for $\epsilon_{\mu \tau}$. Furthermore, our result is complementary to a recent constraint on $\epsilon_{\mu \tau}$ using another publicly available IceCube high-energy event selection. Together, they constitute the world's best limits on nonstandard interactions in the $\mu-\tau$ sector.
\end{abstract}

DOI: 10.1103/PhysRevD.97.072009

\section{INTRODUCTION}

Neutrino flavor change has been observed and confirmed by a plethora of experiments involving solar, atmospheric, reactor, and accelerator-made neutrinos; see [1-3] and references therein. This phenomenon, also known as neutrino oscillations due to its periodic behavior, implies that at least two of the Standard Model (SM) neutrinos have a nonzero mass, making this the first established deviation from the SM. The massive three-neutrino model has been very successful in explaining the neutrino data with two mass differences, known as the solar squared-mass difference $\left(\Delta m_{21}^{2} \approx 7.5 \times 10^{-5} \mathrm{eV}^{2}\right)$ and the atmospheric squaredmass difference $\left(\left|\Delta m_{23}^{2}\right| \approx 2.5 \times 10^{-3} \mathrm{eV}^{2}\right)$ [1,2]. This information, along with the fact that experiments pursuing direct neutrino mass measurements have yielded only upper limits [3], leads to the conclusion that neutrinos have masses that are at least 6 orders of magnitude smaller than those of the charged leptons. Whether these small masses are generated also by the Higgs mechanism, implying the existence of noninteracting right-handed states, or by a different yetunknown mechanism remains an open question.

Many extensions to the SM that incorporate small neutrino masses have been put forward. A subset that addresses small neutrino masses and, at the same time, unifies the electroweak and strong forces is called "Grand Unified Theories" (GUTs). Some of these GUT models predict the existence of heavy $\mathrm{TeV}$-scale bosons [4].

\footnotetext{
*Earthquake Research Institute, University of Tokyo, Bunkyo, Tokyo 113-0032, Japan.

Published by the American Physical Society under the terms of the Creative Commons Attribution 4.0 International license. Further distribution of this work must maintain attribution to the author(s) and the published article's title, journal citation, and DOI. Funded by SCOAP ${ }^{3}$.
}

Searches for direct evidence of these particles have been performed by experiments at the Large Hadron Collider. To date, no evidence has been observed $[5,6]$. In this paper, we address these predictions through a complementary search in the neutrino sector, seeking evidence for new flavorchanging neutrino interactions produced by $\mathrm{TeV}$-scale bosons [7-12].

Nonstandard interactions (NSIs) will introduce modifications of the SM potential, which is relevant for matter effects in neutrino flavor oscillations. The effect of the NSI is expected to grow with distance travelled through matter and becomes more relevant as the neutrino energy increases. As a result, the flux of atmospheric neutrinos detected by the IceCube Neutrino Observatory at the South Pole is ideal for such a study $[9,13]$. In the analysis presented here, we use the data set from [14] obtained with IceCube DeepCore with three years of runtime, which contains multi-GeV atmospheric neutrinos that traverse large fractions of the Earth before reaching the IceCube detector. Because the neutrino production is predominantly from pion and kaon decays, the neutrino flux has wellunderstood initial flavor ratios $[15,16]$.

Current bounds on NSI are reported in [17-19], and current reviews are given in [20-23]. In fact, independent studies of high-energy atmospheric neutrinos using public IceCube data [24] as well as studies with public SuperKamiokande data [25] have already been performed, obtaining strong constraints on NSI parameters. Regarding the latter, the Super-Kamiokande Collaboration has also performed an analysis on NSI parameters [26]. The IceCube studies have so far only used high-energy public data sets, but no low-energy sets. This motivates the presented search, where we focus on the NSI parameter $\epsilon_{\mu \tau}$, which modifies the $\nu_{\mu} \rightarrow \nu_{\tau}$ flavor transition.

The rest of this paper is structured as follows. In Sec. II, we review neutrino oscillations in matter. In Sec. III, we 
describe the NSI theory used in this work. Then, in Sec. IV, we describe the IceCube experiment, and in Sec. V we discuss the main systematics of this analysis. Section VI contains the main results of this paper, and in Sec. VII, we conclude.

\section{MATTER EFFECTS IN NEUTRINO OSCILLATIONS}

Neutrinos are produced in flavor eigenstates but travel as mass eigenstates, meaning that a certain flavor of neutrino produced at the source may later interact as a different flavor [27,28]. At its simplest, when neutrinos travel through vacuum, the oscillation length is given by $L_{\mathrm{osc}}=$ $2.5 \mathrm{~km}(E / \mathrm{GeV})\left(\Delta m^{2} / \mathrm{eV}^{2}\right)^{-1}[3]$.

Since neutrinos interact via neutral- and charged-current weak interactions, neutrino oscillations are modified as matter is traversed. In particular, the propagating neutrino-which is a mixture of electron, muon, and tau flavors-will experience a flavor-dependent matter potential. The relevant potential difference is produced by charged-current coherent forward scattering from electrons in the Earth. We will refer to this as "matter effect," and it is closely related to the Mikheyev-Smirnov-Wolfenstein (MSW) effect $[29,30]$ observed in solar neutrino experiments [31-34]. Indications of matter effects $[35,36]$ in Earth-based oscillation experiments can be extracted from global fits to long-baseline and atmospheric neutrino data sets [37].

\section{NONSTANDARD NEUTRINO INTERACTIONS}

Nonstandard neutrino interactions can be modeled as an additional term in the neutrino Hamiltonian, similar to the conventional matter potential term. The latter effect is included in the neutrino Hamiltonian as a single potential, $V_{C C}$, which modifies the flavor transition probabilities. The potential $\mathrm{V}_{C C}$ is proportional to the Fermi coupling constant $G_{f}$ and the density of electrons $n_{e}$, i.e., $V_{C C}=\sqrt{2} G_{f} n_{e}$.

Adding interactions with nonstandard bosons to the Hamiltonian takes a similar form, but with additional components. To consider all possible flavor-violating interactions, a term $\epsilon_{\alpha \beta}(\alpha, \beta=e, \mu, \tau)$ scales all possible flavor-violating and conserving contributions. For definiteness, in this analysis, we consider nonstandard interactions between neutrinos and down quarks (other assumptions, such as for up quarks, can be approximated by rescaling our results). For this reason, a factor of $n_{d}=3 n_{e}$ (to account for the fact that down-quarks are approximately 3 times as abundant as electrons in the Earth) was used instead of $n_{e}$ as in the case of the SM matter effect. The total Hamiltonian is then

$H_{3 \nu}=\frac{1}{2 E_{\nu}} U M^{2} U^{\dagger}+V_{C C} \operatorname{diag}(1,0,0)+V_{C C} \frac{n_{d}}{n_{e}} \epsilon$, where $E_{\nu}$ is the neutrino energy, $U$ is the neutral lepton mixing matrix (also known as the Pontecorvo-MakiNakagawa-Sakata matrix [27,28]), $M^{2}$ is a diagonal matrix containing the square-mass differences, and

$$
\epsilon=\left(\begin{array}{ccc}
\epsilon_{e e} & \epsilon_{e \mu} & \epsilon_{e \tau} \\
\epsilon_{e \mu}^{*} & \epsilon_{\mu \mu} & \epsilon_{\mu \tau} \\
\epsilon_{e \tau}^{*} & \epsilon_{\mu \tau}^{*} & \epsilon_{\tau \tau}
\end{array}\right),
$$

the NSI strength matrix. Accordingly, the addition of the NSI terms amounts to introducing six additional effective parameters if one accounts for Hermiticity, unitarity constraints, and the possibility of making the Hamiltonian traceless without loss of generality; see [38]. However, for experiments like Super-Kamiokande and IceCube, the terms that correspond to $\nu_{\mu}$ or $\nu_{\tau}$ interactions will dominate. This is because the atmospheric neutrino flux in the $\mathrm{GeV}$ energy range is dominated by $\nu_{\mu}$, which primarily transform into $\nu_{\tau}$ as they travel through the Earth $[39,40]$.

SM matter effects and NSI can be distinguished using the energy and arrival direction distributions of observed flavor-violating transitions. The neutrino flavor oscillations due to the well-established mass differences have been observed from atmospheric neutrinos predominately at energies initially below $10 \mathrm{GeV}$ [41] and recently up to $56 \mathrm{GeV}$ [14]. The observation of atmospheric neutrino oscillations at two different energy ranges but at the same ratio of baseline to energy $(L / E)$ tests the massive three neutrino paradigm and highlights the complementarity of neutrino experiments at different energy ranges. In contrast, the signal predicted for the dominant muon-neutrino to tauneutrino NSI, parametrized by the coupling $\epsilon_{\mu \tau}$, has a smaller magnitude but can be seen over a larger range of energies, as shown in Fig. 1. Therefore, the optimal method for searching for an NSI signal due to $\epsilon_{\mu \tau}$ is to use a large range of neutrino energies, where one expects a combined effect of the NSI and oscillations in the low-energy region and an exclusively NSI signal in the high-energy region. In particular, we note that IceCube's range extends to higher energies than that of previous studies, thus giving us greater sensitivity.

A study by Super-Kamiokande [26], using a twoneutrino approximation, focused on the NSI parameters $\epsilon^{\prime}=\epsilon_{\tau \tau}-\epsilon_{\mu \mu}$ and $\epsilon_{\mu \tau}$. Prior to works using IceCube data, this resulted in the world's best limit with $\left|\epsilon_{\mu \tau}\right|<0.011$ at 90\% C.L. As in the Super-Kamiokande study, we choose to only consider the dominant NSI terms by setting the $\epsilon_{e e}$, $\epsilon_{e \mu}$, and $\epsilon_{e \tau}$ matrix elements to zero, and the Hermiticity of $\epsilon$ is also assumed. Thus, the NSI sector reduces to a two by two matrix, so the $C P$-violating phase can be rephased; i.e., we assume $\epsilon_{\mu \tau}$ to be real. As can be seen in [21], the neutrino mass ordering is degenerate with the sign of $\epsilon_{\mu \tau}$, and the muon neutrino survival probability is symmetric under sign change of $\epsilon^{\prime}$. Given that $\epsilon^{\prime}$ is highly correlated 

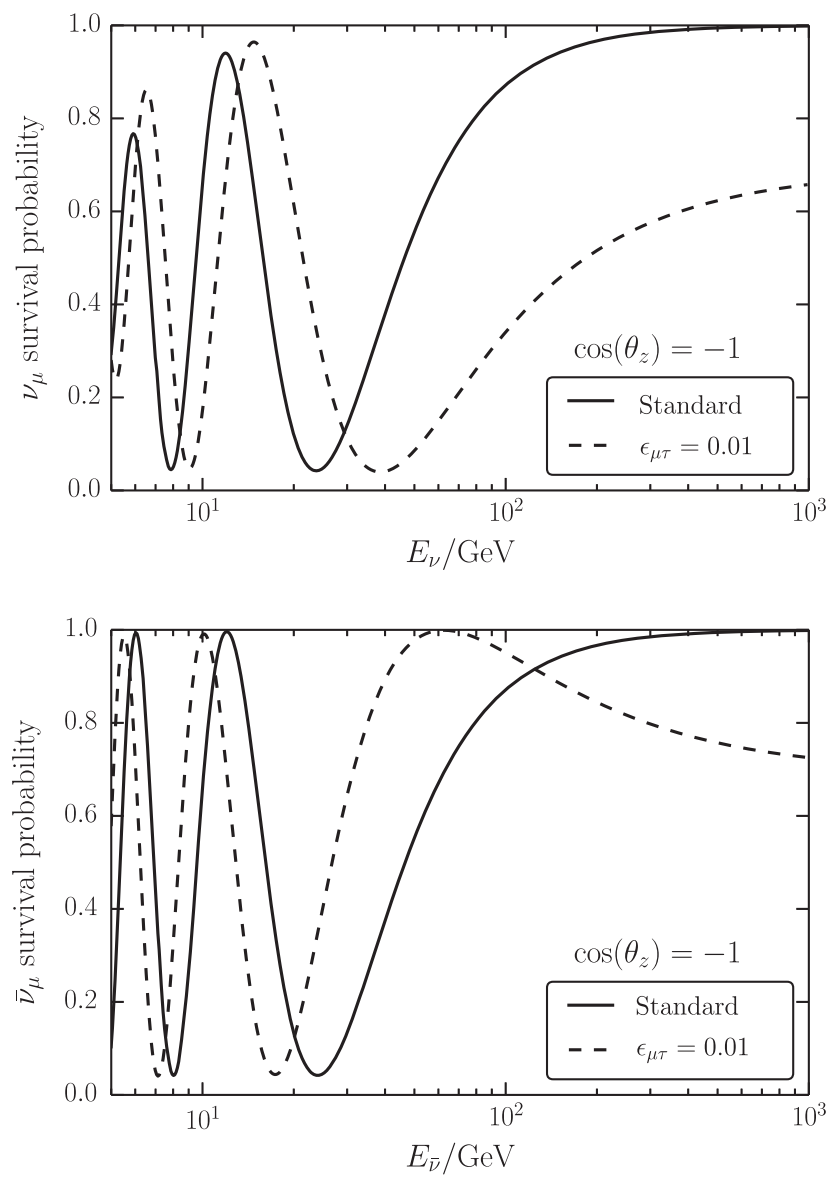

FIG. 1. Muon neutrino (top) and antineutrino (bottom) survival probability at zenith angle $\cos \theta=-1$, corresponding to vertically up going neutrinos that traverse the entire diameter of the Earth, for global best-fit oscillations (solid) and $\epsilon_{\mu \tau}=0.01 \mathrm{NSI}$, close to the current Super-Kamiokande limits (dashed)[26]. NSI effects are visible in the full neutrino energy range of 10-1000 GeV.

with $\epsilon_{\mu \tau}$ in this analysis, we set $\epsilon^{\prime}$ to zero, i.e., $\epsilon_{\tau \tau}=\epsilon_{\mu \mu}$. Also, for definiteness, we assume normal ordering. These assumptions restrict the interpretation of our results $[21,26,42,43]$, as can be seen by simplifying the oscillation expression assuming two-flavor maximal mixing. In this case. the oscillation amplitude is [26]

$$
\sin ^{2} 2 \theta_{\mathrm{eff}}=\frac{\left(1 \pm 2 \eta_{\mu \tau}\right)^{2}}{\left(1 \pm 2 \eta_{\mu \tau}\right)^{2}+\left(\eta^{\prime}\right)^{2}}
$$

where $\eta$ is proportional to $\epsilon$ with proportionality constants that depend on the matter density and $G_{F}$. As expected, when $\epsilon^{\prime}$ becomes large enough, the quantum-Zeno effect [44] applies, and the flavor transitions are suppressed. For large values, this suppresses the well-established standard atmospheric neutrino oscillation, providing constraints on $\epsilon^{\prime}$. On the other hand, null $\epsilon^{\prime}$ provides the strongest constraints on $\epsilon_{\mu \tau}$, which is the scenario we report in this work.

\section{THE ICECUBE DETECTOR}

IceCube is a $1 \mathrm{~km}^{3}$ neutrino detector [45-47] embedded in the ice at the South Pole; see Fig. 2. The detector consists of 86 strings, each with 60 10-inch photomultiplier tubes enclosed in glass spheres, called digital optical modules (DOMs). Of those strings, 78 are separated by a distance of approximately $125 \mathrm{~m}$, with DOMs on each string separated by $17 \mathrm{~m}$. An additional infill extension, DeepCore [48], consists of 8 strings separated by about $75 \mathrm{~m}$, with DOMs on each string separated by $7 \mathrm{~m}$. Secondary particles produced when neutrinos interact in the ice, induce Cherenkov radiation, which is then detected by the DOMs. Muons produce distinctively long tracks. This topology can be reconstructed to determine the angle of the muon with a resolution of $12^{\circ}$ at $10 \mathrm{GeV}$, improving to $6^{\circ}$ at $40 \mathrm{GeV}$ [14]. The energy of the muon can be measured from its track length, while the energy of the hadronic shower produced in the neutrino interaction can be estimated from the total amount of light in the detector. Thus, the muon energy, estimated from the track length, added to the reconstructed shower energy is a proxy for the neutrino energy. The closely spaced DOMs of the DeepCore extension allow measuring the neutrino energy down to neutrino energies of about $5 \mathrm{GeV}$, with a median resolution of $30 \%$ at $8 \mathrm{GeV}$, which improves to $20 \%$ at $20 \mathrm{GeV}$ [14]. This analysis makes use of neutrinos that reach the detector from below the Earth's horizon. This serves two purposes: first it greatly diminishes atmospheric muon contamination and, second, it allows for large matter effects.

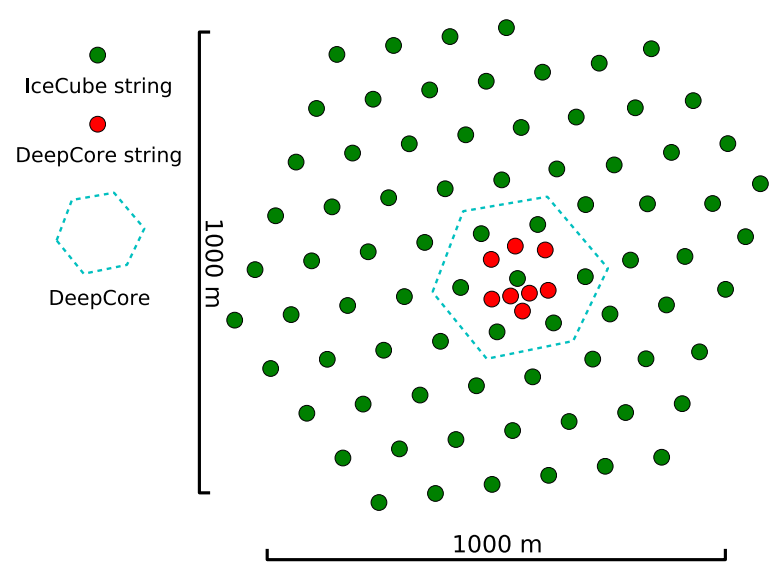

FIG. 2. Detector geometry: green circles represent IceCube strings and red ones DeepCore strings. 


\section{ICECUBE SENSITIVITY TO NONSTANDARD INTERACTIONS AND SYSTEMATIC UNCERTAINTIES}

\section{A. Sensitivity and data set}

IceCube has measured neutrino oscillation parameters by searching for a deficit of neutrinos traveling through Earth and interacting in the detector. In IceCube, the $\nu_{\mu}$ disappearance probability peaks at $\sim 25 \mathrm{GeV}$ for straight up going events, but the oscillation signal is measurable up to about $100 \mathrm{GeV}$, as shown in Fig. 1. In 2014, IceCube published the result of fitting 5174 events from three years of data taken with the complete IceCube detector, obtaining three-neutrino oscillation parameters to a precision comparable with that from dedicated neutrino oscillation experiments [14]. The event selection is based on up going events that interact within DeepCore and follows the procedure in [14]. A high quality of the reconstruction is imposed by requiring that the registered photons are largely unscattered. The atmospheric muon background is rejected efficiently by using the surrounding detector as veto for incoming down-going muons. In addition to the analysis [14], we have improved the background rejection by limiting the amount of charge deposition in the top part of the detector. This sample has approximately $70 \%$ purity of $\nu_{\mu} \mathrm{CC}$ interactions, the rest being dominated by $\mathrm{CC}$ interactions of other flavors and $\mathrm{NC}$ of all flavors with an additional approximately $4 \%$ atmospheric muon contamination.

This study uses a three-neutrino formalism of the neutrino survival probabilities to calculate limits on the $\epsilon_{\mu \tau}$ parameter. We use the publicly available nuSQuIDS neutrino survival probability package $[49,50]$, which has a robust implementation of NSI and uses a detailed Earth density profile [51]. Simulated events are weighted with the Honda et al. atmospheric neutrino model [15], then are binned in an $8 \times 8$ matrix in reconstructed energy, from 6.3 to $56.2 \mathrm{GeV}$, and zenith angle, from $\cos \theta_{z}^{\text {reco }}=-1$ to $\cos \theta_{z}^{\text {reco }}=0$. To determine the expected sensitivity for values of $\epsilon_{\mu \tau}$ in the range of the Super-Kamiokande limit, the total number of events expected with and without NSI effects were calculated as shown in Fig. 3.

This figure shows that the NSI effect increases with increasing $|\cos \theta|$ as a larger amount of matter is traversed; this is also the zenith region where standard oscillations manifest themselves so that interference with them is possible.

\section{B. Systematic uncertainties}

Systematic uncertainties that we have included as nuisance parameters in the fit are

Oscillation parameters: Simultaneously fit for the standard oscillation parameters $\sin ^{2}\left(\theta_{23}\right)$ and $\Delta m_{31}^{2}$ as nuisance parameters.

Ice column scattering coefficient: Scattering of light in the ice that formed within the hole after the DOMs were inserted [52]. This ice contains bubbles that are not found in the bulk ice of the detector. The latter is well studied using flashers and well modeled. The additional bubbles increase the scattering of light, affecting the effective angular efficiency of our DOMs; see [52] for details.

Optical efficiency: The uncertainty in the photon response of the optical modules due to many effects, including photocathode response and obscured regions due to cabling.

Overall normalization $(N)$ : Parameter that scales the event rate expectation freely. This systematic absorbs overall normalization uncertainties due to absolute DOM efficienciy and total cosmic ray flux.

Relative $\nu_{e}$ to $\nu_{\mu}$ normalization $\left(N_{e / \mu}\right)$ : Relative normalization of the electron neutrinos to atmospheric muon neutrinos.

Atmospheric muon fraction $\left(R_{\mu}\right)$ : Normalization of cosmic ray muons that pass the cuts. The distribution
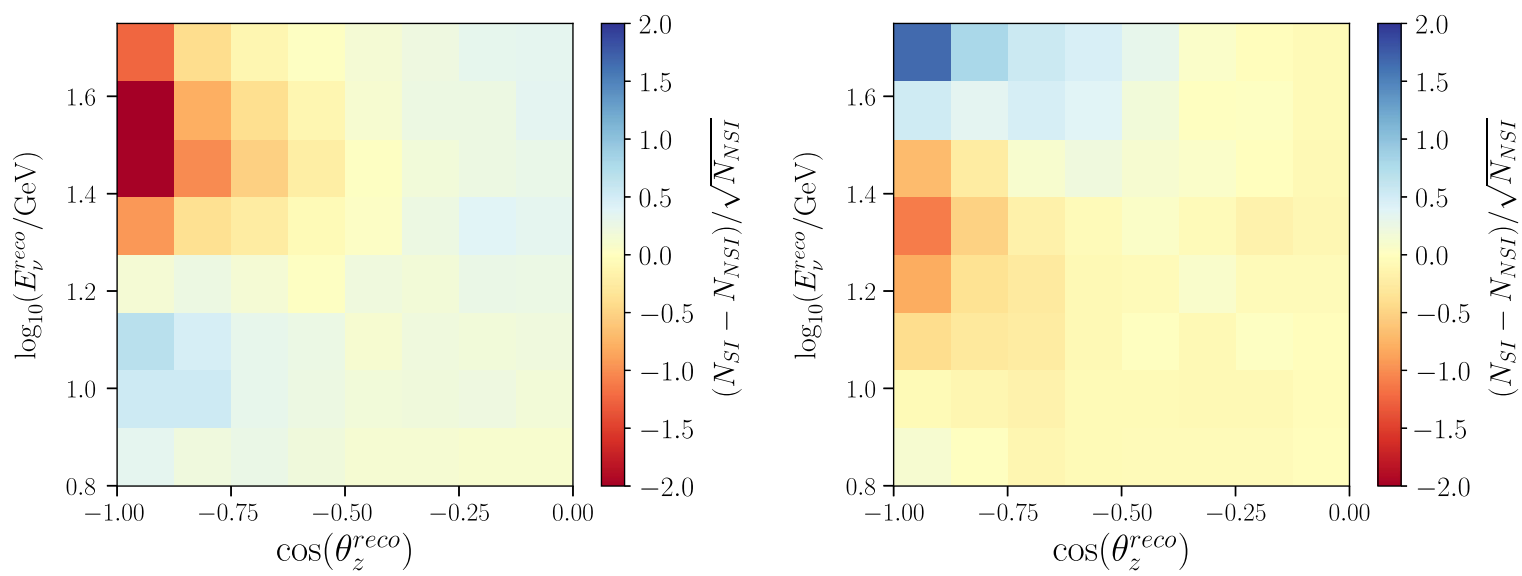

FIG. 3. Expected pulls of predicted event numbers as a function of neutrino energy and zenith angle. The left (right) panel compares $\epsilon_{\mu \tau}=-0.01\left(\epsilon_{\mu \tau}=0.01\right)$ to the standard neutrino oscillation matter effects (SI) expectation. 
of this background was obtained using a data driven method [14].

Spectral index $(\gamma)$ : The exponent describing the energy dependence of the incoming cosmic ray spectrum. This systematic in part accounts for uncertainties due to hadronization processes [53].

For a more detailed discussion of these systematic effects, see [54].

\section{RESULT}

In order to constrain the NSI parameter $\epsilon_{\mu \tau}$, we employ the same data set and event selection in this analysis as was used in [14]. This analysis has the same energy, zenith angle resolution, and systematic uncertainties as the analyses in [14,54] with an additional fiducial volume cut, resulting in a final sample of 4625 events [54]. To determine the best-fit oscillation parameters, the simulated data distributions are compared to the data bin-by-bin.
Minimizing the Poisson likelihood value of the data given the Monte Carlo, modified by the nuisance parameters (as described in $[14,54])$, determines the final best-fit parameters. The data and Monte Carlo at the best-fit point are in good agreement after the fit, as shown in Fig. 4, with bestfit nuisance parameters given in Table I. The 90\% C.L. limits are then calculated using the difference from the bestfit likelihood, assuming Wilks's theorem applies [55]. We obtain a likelihood that would correspond to a $\chi^{2} /$ dof $=53.82 / 55$. To make the comparison to [24], we also calculate the credibility regions by integrating the profiled likelihood using a uniform prior on $\epsilon_{\mu \tau}$ and profiling over the nuisance parameters. This procedure is found to be in good agreement with the result obtained using Wilks's theorem.

The resulting constraints on the NSI parameters are shown in Fig. 5, with the best-fit values for the systematic parameters shown in Table I. Priors on the atmospheric and

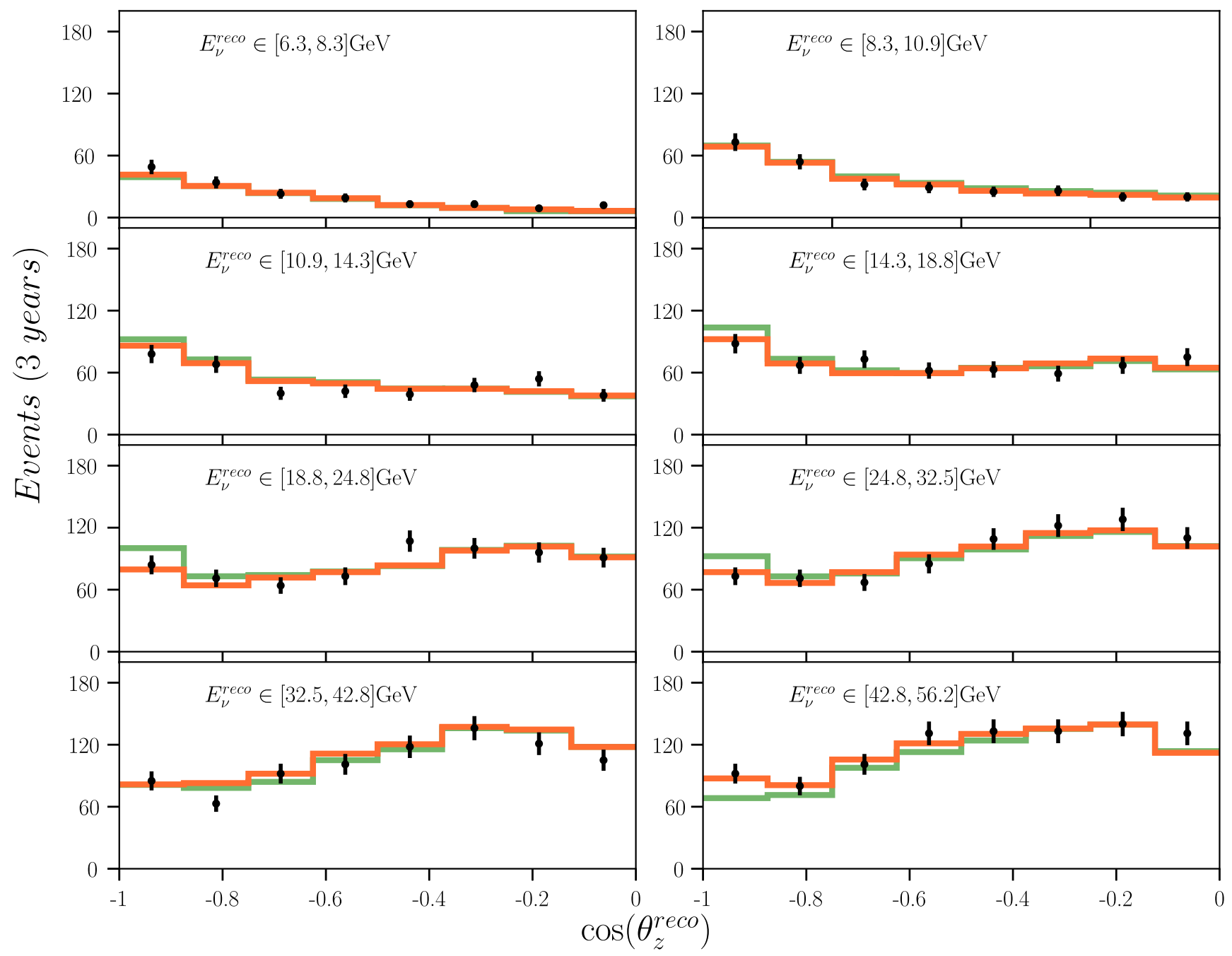

FIG. 4. Data (black points) and Monte Carlo (solid lines) comparisons for the best-fit nuisance parameters of this analysis, as a function of the arrival direction, $\cos \left(\theta_{z}^{\text {reco }}\right)$, in the eight different energy bins. The orange color corresponds to the best-fit point $\left(\epsilon_{\mu \tau}=-5 \times 10^{-4}\right)$ and the light green to $\epsilon_{\mu \tau}=0.015$. The error bars include statistical uncertainties only. 
TABLE I. List of the best-fit parameters obtained in this analysis. When priors are listed, they are Gaussian, and the width corresponds to the one sigma range. Values obtained at the best-fit point are also listed.

\begin{tabular}{lccc}
\hline \hline Parameter & $\begin{array}{c}\text { Prior } \\
\text { center }\end{array}$ & $\begin{array}{c}\text { Prior } \\
\text { width }\end{array}$ & Fit \\
\hline$\epsilon_{\mu \tau}$ & $\ldots$ & $\ldots$ & -0.0005 \\
$\sin ^{2}\left(\theta_{23}\right)$ & $\ldots$ & $\ldots$ & 0.52 \\
$\Delta m_{31}^{2} / 10^{-3} \mathrm{eV}^{2}$ & $\ldots$ & $\ldots$ & 2.62 \\
Ice column scattering $\left(\mathrm{cm}^{-1}\right)$ & 0.02 & 0.01 & 0.02 \\
Optical efficiency $(\%)$ & 100 & 10 & 101 \\
Overall normalization $(N)$ & $\ldots$ & $\ldots$ & 1.00 \\
Relative $\nu_{e}$ normalization $\left(N_{e / \mu}\right)$ & 1 & 0.20 & 1.03 \\
Atmospheric $\mu\left(R_{\mu}\right)$ & $\ldots$ & $\ldots$ & 0.0 \\
Spectral index $(\gamma)$ & 2.70 & 0.05 & 2.67 \\
\hline \hline
\end{tabular}

detector nuisance parameters are the same as in [14]. Furthermore, Fig. 6 shows the correlation between the fit parameters at the best fit of oscillation and nuisance parameters. The mass-squared difference $\Delta m_{31}^{2}$ exhibits the strongest correlation with $\epsilon_{\mu \tau}$. This is to be expected from existing correlations and degeneracies in the oscillation probability [38]. Finally, the change in the oscillation parameters compared to [14] has been demonstrated to be caused by the additional cut on the fiducial volume.

For this analysis, the best fit is at $\epsilon_{\mu \tau}=-0.0005$. The $90 \%$ C.L. range is $-0.0067<\epsilon_{\mu \tau}<0.0081$. This result is consistent with the Super-Kamiokande limits for $\epsilon_{\mu \tau}$ [26] and represents an independent determination of the

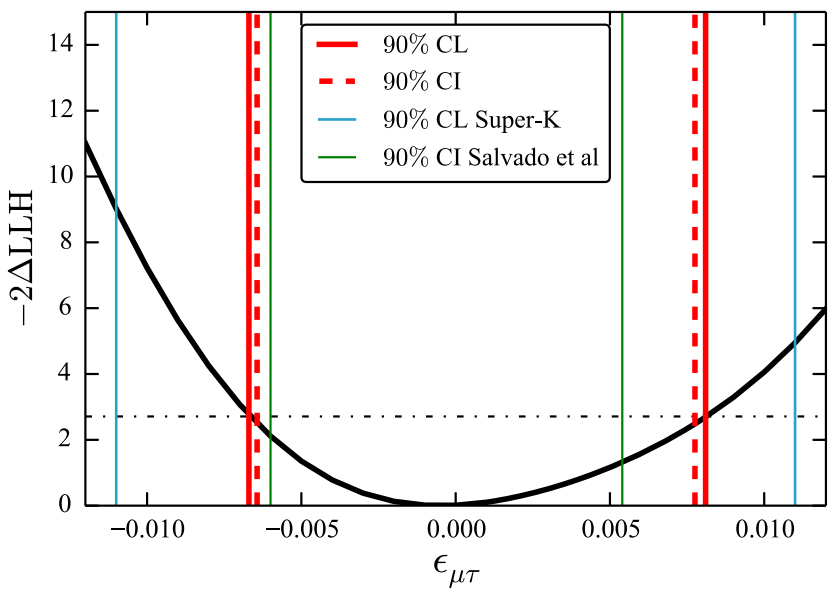

FIG. 5. Confidence limits from this analysis on the NSI parameter $\epsilon_{\mu \tau}$ using the event selection from [14,54] shown as solid vertical red lines. Similarly, dashed vertical red lines show the $90 \%$ credibility interval using a flat prior on $\epsilon_{\mu \tau}$ and where we have profiled over the nuisance parameters. The light blue vertical lines show the Super-Kamiokande 90\% C.L. [26]. The light green lines show the $90 \%$ credibility region from [24]. Finally, the horizontal dash-dot line indicates the value of $-2 \Delta \mathrm{LLH}$ that corresponds to a $90 \%$ confidence interval according to Wilks's theorem.

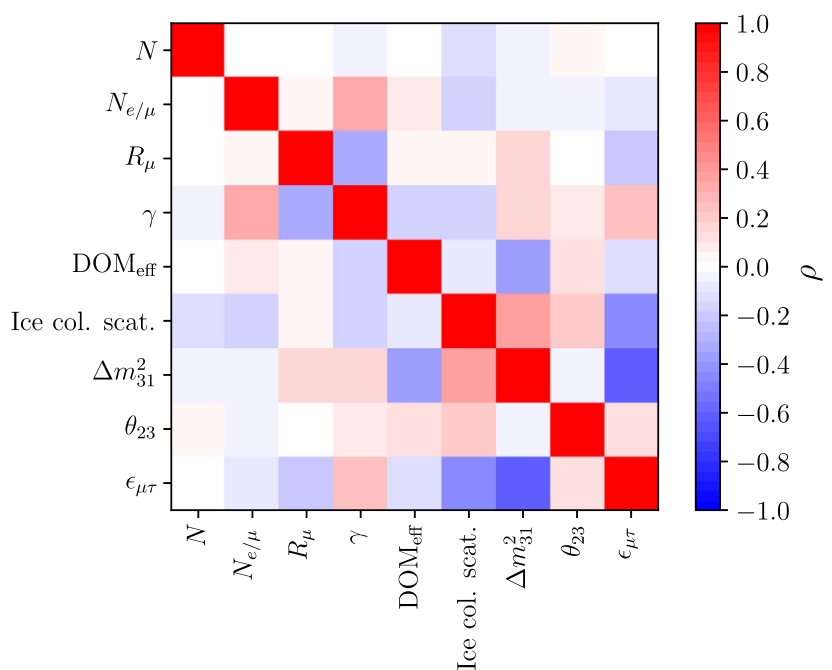

FIG. 6. Correlation matrix of the nuisance and physics parameters considered in this analysis calculated at the maximum likelihood solution. The color scale shows the correlation coefficient $(\rho)$.

parameter. To compare with this, in Fig. 5 we show the results from [24] obtained using public IceCube highenergy data. Fig. 1 shows that the signal for $\epsilon_{\mu \tau}$ is largest in the region above $100 \mathrm{GeV}$. A planned extension of this study including a sample of events above $100 \mathrm{GeV}$ would significantly improve constraints on NSI parameters [13].

\section{CONCLUSIONS}

The existence of physics beyond the standard model has been suggested by the nonzero neutrino mass, in addition to the existence of dark matter. Extensions of the standard model that explain these observations could lead to a modified strength of neutrino interactions in standard matter. Experiments like IceCube have the potential to constrain these nonstandard interactions with greater precision than previous experiments.

Our best fit of the NSI flavor-changing parameter yields $\epsilon_{\mu \tau}=-0.0005$, with a $90 \%$ C.L. range of $-0.0067<\epsilon_{\mu \tau}<0.0081$. This result is comparable to, and provides a slight improvement over, the SuperKamiokande limits for $\epsilon_{\mu \tau}\left(\left|\epsilon_{\mu \tau}\right|<0.011\right.$ at $90 \%$ C.L.). A recent study [24] using IceCube public data obtained constraints which are slightly better than the ones shown in this paper. These constraints are also shown in Fig. 5 and are complementary to our result as they are affected by different systematics and make use of a different energy regime.

\section{ACKNOWLEDGMENTS}

The IceCube Collaboration designed, constructed and now operates the IceCube Neutrino Observatory. Data processing and calibration, Monte Carlo simulations of 
the detector and of theoretical models, and data analyses were performed by a large number of collaboration members, who also discussed and approved the scientific results presented here. The main authors of this manuscript were M. Day, C. Argüelles, and J. M. Conrad. It was reviewed by the entire collaboration before publication, and all authors approved the final version of the manuscript. We acknowledge the support from the following agencies: United States-U.S. National Science Foundation-Office of Polar Programs, U.S. National Science Foundation-Physics Division, Wisconsin Alumni Research Foundation, Center for High Throughput Computing (CHTC) at the University of WisconsinMadison, Open Science Grid (OSG), Extreme Science and Engineering Discovery Environment (XSEDE), U.S. Department of Energy-National Energy Research Scientific Computing Center, Particle Astrophysics Research Computing Center at the University of Maryland, Institute for Cyber-Enabled Research at Michigan State University, and Astroparticle Physics Computational Facility at Marquette University; Belgium-Funds for Scientific Research (FRS-FNRS and FWO), FWO Odysseus and
Big Science programs, and Belgian Federal Science Policy Office (Belspo); Germany-Bundesministerium für Bildung und Forschung (BMBF), Deutsche Forschungsgemeinschaft (DFG), Helmholtz Alliance for Astroparticle Physics (HAP), Initiative and Networking Fund of the Helmholtz Association, Deutsches Elektronen Synchrotron (DESY), and High Performance Computing cluster of the RWTH Aachen; Sweden-Swedish Research Council, Swedish Polar Research Secretariat, Swedish National Infrastructure for Computing (SNIC), and Knut and Alice Wallenberg Foundation; Australia-Australian Research Council; Canada-Natural Sciences and Engineering Research Council of Canada, Calcul Qubec, Compute Ontario, Canada Foundation for Innovation, WestGrid, and Compute Canada; Denmark-Villum Fonden, Danish National Research Foundation (DNRF); New Zealand-Marsden Fund; Japan-Japan Society for Promotion of Science (JSPS) and Institute for Global Prominent Research (IGPR) of Chiba University; KoreaNational Research Foundation of Korea (NRF); Switzerland-Swiss National Science Foundation (SNSF).
[1] I. Esteban, M. C. Gonzalez-Garcia, M. Maltoni, I. MartinezSoler, and T. Schwetz, Updated fit to three neutrino mixing: exploring the accelerator-reactor complementarity, J. High Energy Phys. 01 (2017) 087.

[2] F. Capozzi, E. Lisi, A. Marrone, D. Montanino, and A. Palazzo, Neutrino masses and mixings: Status of known and unknown $3 \nu$ parameters, Nucl. Phys. B908, 218 (2016).

[3] C. Patrignani et al. (Particle Data Group), Review of particle physics, Chin. Phys. C 40, 100001 (2016).

[4] S. F. King, Unified models of neutrinos, flavour and $C P$ violation, Prog. Part. Nucl. Phys. 94, 217 (2017).

[5] G. Aad et al. (ATLAS Collaboration), Search for high-mass diboson resonances with boson-tagged jets in proton-proton collisions at $\sqrt{s}=8 \mathrm{TeV}$ with the ATLAS detector, J. High Energy Phys. 12 (2015) 055.

[6] V. Khachatryan et al. (CMS Collaboration), Search for massive resonances in dijet systems containing jets tagged as $\mathrm{W}$ or $\mathrm{Z}$ boson decays in pp collisions at $\sqrt{s}=8 \mathrm{TeV}$, J. High Energy Phys. 08 (2014) 173.

[7] S. Weinberg, Baryon and Lepton Nonconserving Processes, Phys. Rev. Lett. 43, 1566 (1979).

[8] N. Fornengo, M. Maltoni, R. Tomas, and J. W. F. Valle, Probing neutrino nonstandard interactions with atmospheric neutrino data, Phys. Rev. D 65, 013010 (2001).

[9] M. C. Gonzalez-Garcia and M. Maltoni, Atmospheric neutrino oscillations and new physics, Phys. Rev. D 70, 033010 (2004).

[10] J. Kopp, M. Lindner, T. Ota, and J. Sato, Non-standard neutrino interactions in reactor and superbeam experiments, Phys. Rev. D 77, 013007 (2008).
[11] S. Antusch, J. P. Baumann, and E. Fernandez-Martinez, Non-standard neutrino interactions with matter from physics beyond the standard model, Nucl. Phys. B810, 369 (2009).

[12] A. M. Gago, H. Minakata, H. Nunokawa, S. Uchinami, and R. Zukanovich Funchal, Resolving $C P$ violation by standard and nonstandard interactions and parameter degeneracy in neutrino oscillations, J. High Energy Phys. 01 (2010) 49.

[13] A. Esmaili and A. Y. Smirnov, Probing non-standard interaction of neutrinos with IceCube and DeepCore, J. High Energy Phys. 06 (2013) 026.

[14] M. G. Aartsen et al. (IceCube Collaboration), Determining neutrino oscillation parameters from atmospheric muon neutrino disappearance with three years of IceCube DeepCore data, Phys. Rev. D 91, 072004 (2015).

[15] M. Honda, M. Sajjad Athar, T. Kajita, K. Kasahara, and S. Midorikawa, Atmospheric neutrino flux calculation using the NRLMSISE-00 atmospheric model, Phys. Rev. D 92, 023004 (2015).

[16] M. Honda, T. Kajita, K. Kasahara, and S. Midorikawa, Improvement of low energy atmospheric neutrino flux calculation using the JAM nuclear interaction model, Phys. Rev. D 83, 123001 (2011).

[17] C. Biggio, M. Blennow, and E. Fernandez-Martinez, General bounds on non-standard neutrino interactions, J. High Energy Phys. 08 (2009) 090.

[18] C. Biggio, M. Blennow, and E. Fernandez-Martinez, Loop bounds on non-standard neutrino interactions, J. High Energy Phys. 03 (2009) 139. 
[19] F. J. Escrihuela, O. G. Miranda, M. A. Tortola, and J. W. F. Valle, Constraining nonstandard neutrino-quark interactions with solar, reactor and accelerator data, Phys. Rev. D 80, 105009 (2009); Erratum, Phys. Rev. D 80, 129908 (2009).

[20] T. Ohlsson, Status of non-standard neutrino interactions, Rep. Prog. Phys. 76, 044201 (2013).

[21] O. G. Miranda and H. Nunokawa, Non standard neutrino interactions: Current status and future prospects, New J. Phys. 17, 095002 (2015).

[22] S. Davidson, C. Pena-Garay, N. Rius, and A. Santamaria, Present and future bounds on nonstandard neutrino interactions, J. High Energy Phys. 03 (2003) 011.

[23] F. J. Escrihuela, M. Tortola, J. W. F. Valle, and O. G. Miranda, Global constraints on muon-neutrino non-standard interactions, Phys. Rev. D 83, 093002 (2011).

[24] J. Salvado, O. Mena, S. Palomares-Ruiz, and N. Rius, Nonstandard interactions with high-energy atmospheric neutrinos at IceCube, J. High Energy Phys. 01 (2017) 141.

[25] S. Fukasawa and O. Yasuda, Constraints on the nonstandard interaction in propagation from atmospheric neutrinos, Adv. High Energy Phys. 2015, 1 (2015).

[26] G. Mitsuka et al. (Super-Kamiokande Collaboration), Study of non-standard neutrino interactions with atmospheric neutrino data in Super-Kamiokande I and II, Phys. Rev. D 84, 113008 (2011).

[27] B. Pontecorvo, Neutrino experiments and the problem of conservation of leptonic charge, Zh. Eksp. Teor. Fiz. 53, 1717 (1967) [Sov. Phys. JETP 26, 984 (1968)].

[28] Z. Maki, M. Nakagawa, and S. Sakata, Remarks on the unified model of elementary particles, Prog. Theor. Phys. 28, 870 (1962).

[29] L. Wolfenstein, Neutrino oscillations in matter, Phys. Rev. D 17, 2369 (1978).

[30] S. P. Mikheev and A. Y. Smirnov, Resonance amplification of oscillations in matter and spectroscopy of solar neutrinos, Yad. Fiz. 42, 1441 (1985) [Sov. J. Nucl. Phys. 42, 913 (1985)].

[31] K. Abe et al. (Super-Kamiokande Collaboration), Solar neutrino results in Super-Kamiokande-III, Phys. Rev. D 83, 052010 (2011).

[32] Q. R. Ahmad et al. (SNO Collaboration), Measurement of the Rate of $\nu_{e}+d \rightarrow p+p+e^{-}$Interactions Produced by ${ }^{8} B$ Solar Neutrinos at the Sudbury Neutrino Observatory, Phys. Rev. Lett. 87, 071301 (2001).

[33] B. Aharmim et al. (SNO Collaboration), Combined analysis of all three phases of solar neutrino data from the Sudbury Neutrino Observatory, Phys. Rev. C 88, 025501 (2013).

[34] G. Bellini et al., Precision Measurement of the $\mathrm{Be}^{7}$ Solar Neutrino Interaction Rate in Borexino, Phys. Rev. Lett. 107, 141302 (2011).

[35] M. Freund, M. Lindner, S. T. Petcov, and A. Romanino, Testing matter effects in very long baseline neutrino oscillation experiments, Nucl. Phys. B578, 27 (2000).

[36] E. K. Akhmedov, A. Dighe, P. Lipari, and A. Y. Smirnov, Atmospheric neutrinos at Super-Kamiokande and paramet- ric resonance in neutrino oscillations, Nucl. Phys. B542, 3 (1999).

[37] M. C. Gonzalez-Garcia and M. Maltoni, Determination of matter potential from global analysis of neutrino oscillation data, J. High Energy Phys. 09 (2013) 152.

[38] J. Liao, D. Marfatia, and K. Whisnant, Degeneracies in long-baseline neutrino experiments from nonstandard interactions, Phys. Rev. D 93, 093016 (2016).

[39] E. K. Akhmedov, Neutrino physics, in Proceedings, Summer School in Particle Physics: Trieste, Italy, 1999 (1999), p. 103.

[40] M. Fukugita and T. Yanagida, Physics of Neutrinos and Applications to Astrophysics (Springer, Berlin, 2003).

[41] Y. Fukuda et al. (Super-Kamiokande Collaboration), Evidence for Oscillation of Atmospheric Neutrinos, Phys. Rev. Lett. 81, 1562 (1998).

[42] M. C. Gonzalez-Garcia, M. Maltoni, and J. Salvado, Testing matter effects in propagation of atmospheric and longbaseline neutrinos, J. High Energy Phys. 05 (2011) 075.

[43] P. Coloma and T. Schwetz, Generalized mass ordering degeneracy in neutrino oscillation experiments, Phys. Rev. D 94, 055005 (2016).

[44] B. Misra and E. C. G. Sudarshan, The zeno's paradox in quantum theory, J. Math. Phys. (N.Y.) 18, 756 (1977).

[45] A. Achterberg et al. (IceCube Collaboration), First year performance of The IceCube Neutrino Telescope, Astropart. Phys. 26, 155 (2006).

[46] R. Abbasi et al. (IceCube Collaboration), The IceCube Data Acquisition System: Signal capture, digitization, and timestamping, Nucl. Instrum. Methods Phys. Res., Sect A 601, 294 (2009).

[47] M. G. Aartsen et al. (IceCube Collaboration), The IceCube Neutrino Observatory: Instrumentation and online systems, J. Instrum. 12, P03012 (2017).

[48] R. Abbasi et al., The design and performance of IceCube DeepCore, Astropart. Phys. 35, 615 (2012).

[49] C. A. Argüelles Delgado, J. Salvado, and C. N. Weaver, A simple quantum integro-differential solver (SQuIDS), Comput. Phys. Commun. 196, 569 (2015).

[50] C. A. Argüelles, J. Salvado, and C. N. Weaver, $\nu$-SQuIDS, https://github.com/Arguelles/nuSQuIDS (2015).

[51] A. M. Dziewonski and D. L. Anderson, Preliminary reference Earth model, Phys. Earth Planet. Interiors 25, 297 (1981).

[52] M. G. Aartsen et al. (IceCube Collaboration), Measurement of South Pole ice transparency with the IceCube LED calibration system, Nucl. Instrum. Methods Phys. Res., Sect. A 711, 73 (2013).

[53] T. Katori and S. Mandalia, PYTHIA hadronization process tuning in the GENIE neutrino interaction generator, J. Phys. G 42, 115004 (2015).

[54] M. G. Aartsen et al. (IceCube Collaboration), Search for sterile neutrino mixing using three years of IceCube DeepCore data, Phys. Rev. D 95, 112002 (2017).

[55] S. S. Wilks, The large-sample distribution of the likelihood ratio for testing composite hypotheses, Ann. Math. Stat. 9, 60 (1938). 